\title{
Evaluation of Used-Parts Quality for Integration of the Inverse Manufacturing Process*
}

\author{
Michiko Matsuda ${ }^{* 1}$, Hiroto Suzuki ${ }^{* 2}$ and Fumihiko Kimura ${ }^{* 3}$ \\ ${ }^{*}$ Department of Information and Computer Sciences, Kanagawa Institute of Technology \\ 1030 Shimo-ogino, Atsugi-shi, Kanagawa 243-0292, Japan \\ telephone:+81-462-91-3213, fax:+81-462-42-8490, e-mail: matsuda@ic.kanagawa-it.ac.jp \\ ${ }^{*}$ Arthur D. Little (Japan), Inc. \\ ${ }^{*}$ Graduate School of Engineering, The University of Tokyo
}

\begin{abstract}
To complete the product life cycle, one has to include an inverse flow from products to materials or parts. In the inverse manufacturing process, products are decomposed into several parts after the use phase. Some of these parts are re-used at the production stage and during the maintenance/up-grade process. In this paper, the evaluation system of decomposed parts' quality is proposed in order to connect the output from the inverse process with the input to the forward manufacturing process. To represent the deteriorated status of used parts, geometrical quality description is introduced into the product model. Behavior-mechanism relation tables are used for connecting functional behavior of the product with corresponding deteriorating parts in the evaluation process. Final output from the evaluation process is geometrical deteriorated status of the geometric element of the parts. These data can be used at planning the stage for reusing parts in the forward flow of product life cycle.
\end{abstract}

Key words: computer-aided integrated manufacturing, inverse manufacturing, product life cycle, product modeling, used parts quality evaluation

\section{INTRODUCTION}

An active discussion on product life cycle has recently ensued, driven by an increasing interest in the environmental impact of manufacturing. Product design is the initial stage of the product life cycle. The product design

\footnotetext{
* Funding support: Grant-in-Aid of Scientific Research (C) by The Ministry of Education, Science, Sports and Culture (Japan)

The original version of this chapter was revised: The copyright line was incorrect. This has been corrected. The Erratum to this chapter is available at DOI: 10.1007/978-0-387-35492-7_50
} 
process has to be supported with considerations of environmental aspects. Furthermore, an approach is now made to not only consider the product design and manufacturing preparation concurrently, but also to design the total product life cycle as a whole from product planning, through product design and manufacturing, to product usage, maintenance and reuse/recycling/disposal. This approach is called "inverse manufacturing", stressing the controllability of the re-use/recycling process, where closed cycles of manufacturing activity are pre-planned and controlled $[1,2]$.

The trends mentioned above demand the necessity to make the production process more energy efficient as well as to save resources. They require that the manufacturing preparation system should estimate not only the production time and cost but also energy usage, and incorporate the reuse/recycle of used-parts and materials wherever possible, and so on.

In this paper, mechanical products are considered that are produced with large varieties of types and very small production volumes. In this type of production, it is important that used parts from a product can be accepted as materials for another product in the manufacturing phase [3]. Therefore, it is important to support the reuse phase by constructing the additional description of representing the deteriorated status in the product model and by providing the evaluation method for the geometrical quality of each part using this model. As a result, this support connects the inverse flow with the forward flow in the product life cycle and completes the product life cycle lines.

\section{INVERSE / FORWARD PROCESS INTEGRATION}

\subsection{Life cycles of product}

The life cycle of a certain product includes various phases such as product design, manufacturing, usage/maintenance, disassembly and reuse/disposal. In these phases, the reuse phase can be divided into two types. One is the reuse of each part. In this type of reuse, decomposed parts are refurbished and/or exposed to another machining process in order to adjust their form and accuracy to a new usage. The other type of reuse is material recycling by melting down parts. In this paper, only the former type of reuse for each part is considered.

As seen from the manufacturing phase, various kinds of products originating from various and separate life cycles are processed on one common production line. Figure 1 shows this. To represent a complete product life cycle, one has to include an inverse flow from products to parts. In the inverse process, products are decomposed into several parts after the use phase. Some of these parts are reused at the manufacturing stage and 
during the maintenance/up-grade process.

In the manufacturing phase considering parts reuse, several types of parts from different products have to be processed in the same manufacturing process. The manufacturing process can be divided into machining process and assembly process. Many of the mechanical products, which are considered in this paper, have already been intended to be used repeatedly by refurbishment if necessary [4].

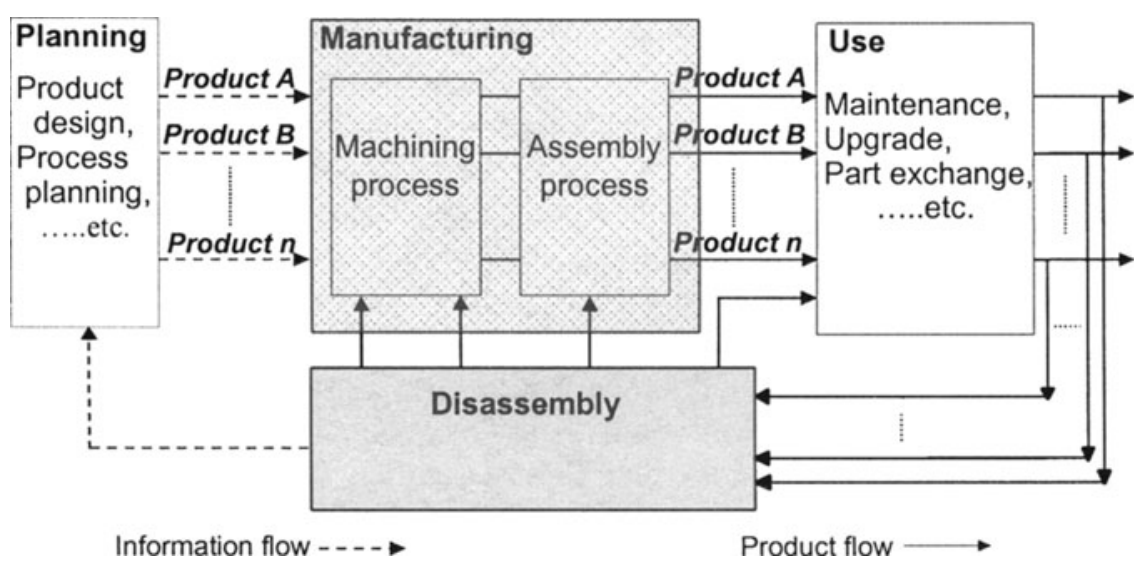

Figure 1. Product life cycle and Inverse manufacturing

\subsection{Introduction of evaluation process}

After the usage/maintenance stage, the product is disassembled into several parts in the inverse manufacturing process. By reusing these parts, the inverse process is integrated into the traditional forward manufacturing process. If only blank materials are used in the manufacturing process, only one machining process plan can be designed. However, if used parts from different products are also treated as candidates of machining in a material stock, several processes should be considered. Because the deteriorated status of each used part can be different, one part may need to be refurbished while others may be able to be used as assembly parts without refurbishment. Also some parts may be used as repair parts in the maintenance process. Here, refurbishment means the additional machining required for remaking the parts into the parts required for another product. Figure 2 shows this integration.

When reusing used parts, their quality needs to be evaluated based on their usage history data, since used parts may have changed their size and accuracy due to deterioration such as abrasion and deformation during their 
former use. In order to support this evaluation process from the product information processing, the structure of the product model and the new evaluation procedure should be provided.

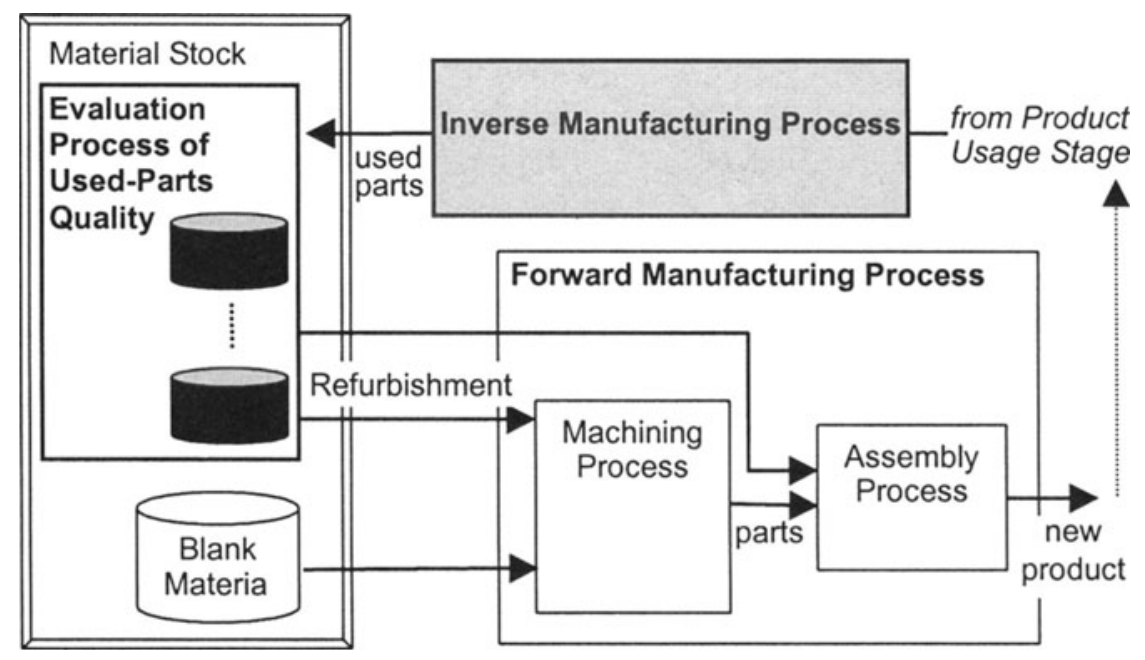

Figure 2. Integration of inverse manufacturing process and traditional process

\section{PRODUCT MODEL FOR EVALUATION}

\subsection{Role of product model in evaluation process}

For reusing parts, the product model has to be able to be used in the quality evaluation of used parts before their reuse. For this evaluation, the product model has to fulfil the following three requirements [5].

The first requirement is the ability to apply the prediction process of the geometrical deteriorated status using a computational method such as a behavior simulation. As considered, a geometry-oriented product model is mainly applied to predict how a certain geometrical property, such as accuracy of form may have an effect on the product behavior. In the product model, these geometrical accuracies are represented in the geometric model, and should be treated as parameters of the input model in the calculation process.

The second requirement is the ability to add the result of the evaluation into the original product model. In the present product model, it is hardly supposed that the information about geometrical quality should be added for the inverse process. In order to add the result of the evaluation process, 
another attribute should be added to the normal product model.

The third requirement is the ability to be used for production planning including refurbishment. Since such machining is done for each part, these geometrical accuracies have to be assigned to each geometric element from the refurbishment viewpoint. The deteriorated condition of each used part is evaluated as a change in accuracy of form based on the usage history. An altered product model based on the evaluation of deterioration is added to a material library as a candidate for machining. If it matches requirements for the parts of another product, refurbishing process data for the selected candidate parts are generated based on the selected product model. Then this product model is again circulated as a new product model in the next life cycle.

\subsection{Geometrical quality description}

The geometrical quality description is to relate the mechanical behaviors and the geometrical accuracies. Figure 3 shows the structure of geometrical quality description [5].

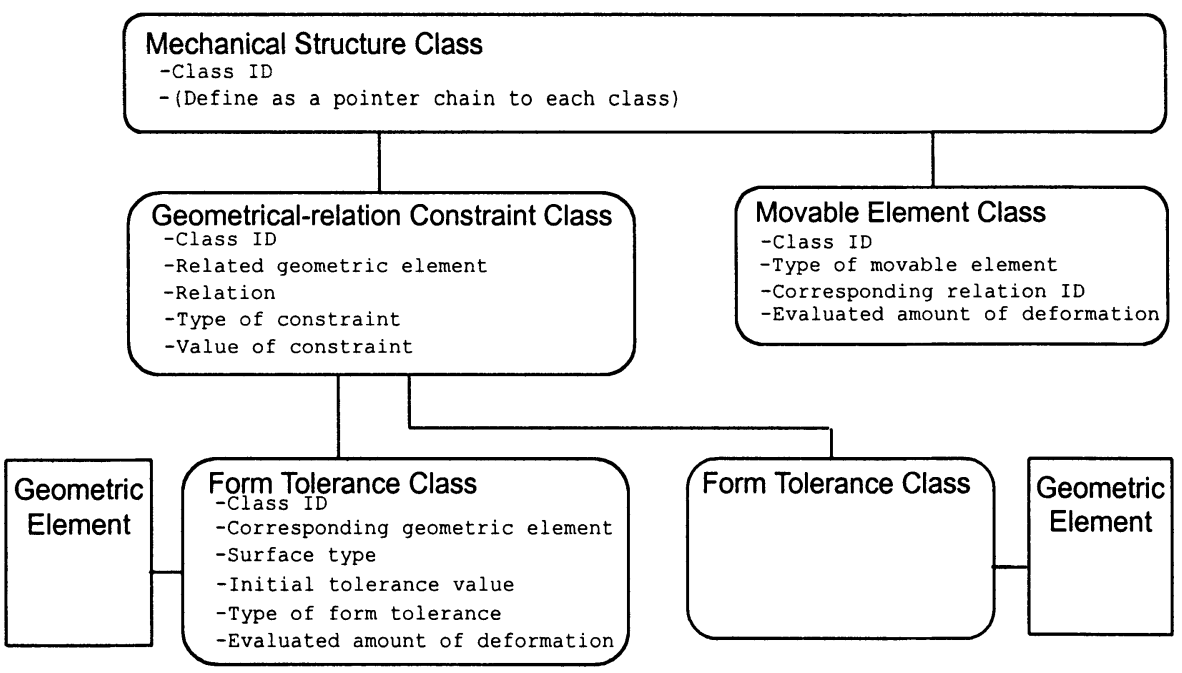

Figure 3. Geometrical quality description

Form tolerances are defined for each geometric element. The type of form tolerance is determined according to the surface type of the geometric element. A geometrical-relation constraint is defined for the geometrical relation between geometric elements. If the geometrical-relation constraint is defined between parts and any relative motion occurs in the relation, a movable element has also to be defined for the geometrical-relation 
constraint. As commonly used movable element descriptions have already been constructed, the appropriate movable element is applied to the objective movable relation. The whole connection between each description in the product is described as a mechanical structure description. In this description, the connective relation is represented in the form of a pointer chain between each description.

\subsection{Product description for evaluation}

In the mechanical product, many of the product functions are realized through the mechanical behaviors. These behaviors are made up of the mechanical capability of each part as well as the part's role in the overall structure. Therefore the geometrical accuracy given by the geometrical variations of each part and its relation can have an effect on the behavior.

Figure 4 shows the additional description required in the product model for evaluating used parts' quality. Functional constraints for satisfying the product function are defined as the geometrical accuracies of assembly relations, non-interference with the motion envelope of moving parts, and so on.

Corresponding to each functional constraint, behavior-mechanism relation tables are generated from the product model. In this table, the possible sets of the movable elements in which deteriorations occur are given corresponding to a functional constraint to be achieved.

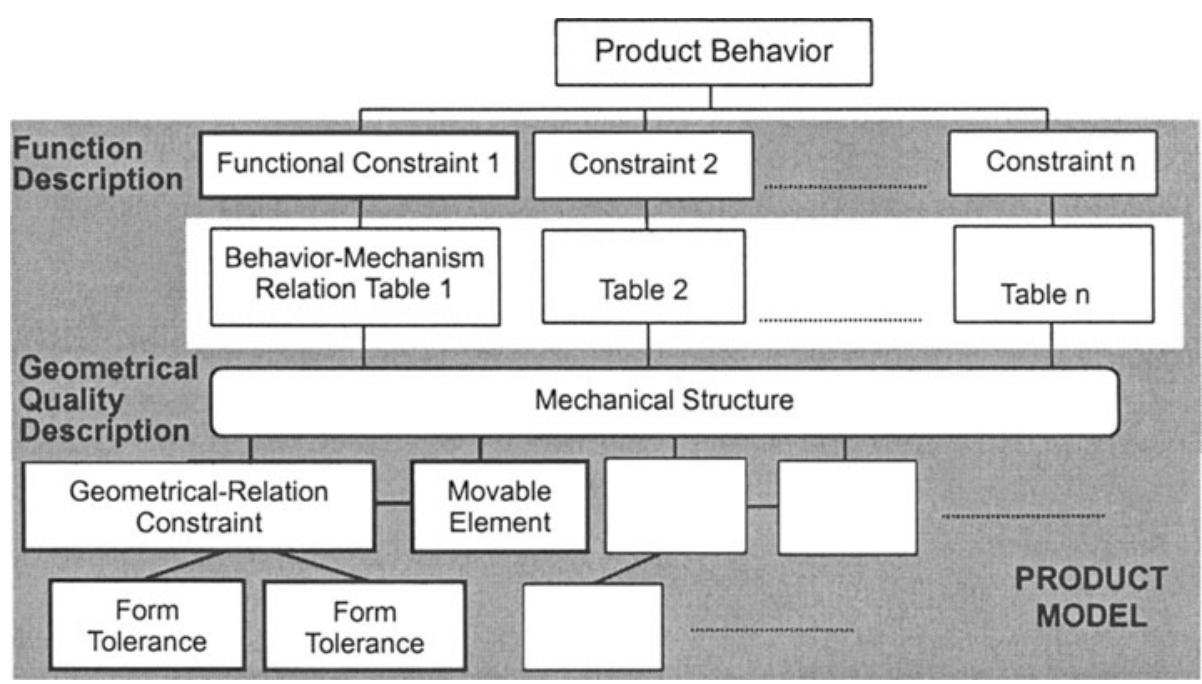

Figure 4. Product description for evaluation of used-parts quality 


\section{EVALUATION OF USED-PARTS QUALITY}

\subsection{Evaluation process}

The evaluation process for geometrical quality of used parts has two phases. The first phase is the preparation of the behavior-mechanism relation table, and the other phase is execution of the evaluation. The Evaluation process using the product model is shown in Figure 5.

Usually, the behavior-mechanism relation tables can be prepared at the product design stage. The behavior-mechanism relation tables are generated corresponding to each functional constraint. The value of the corresponding predicted geometrical accuracy in the behavior-mechanism relation tables are obtained from the product mechanical-behavior simulation.

At the execution phase of the evaluation, the product behavior at the product usage stage is compared with functional constraints which the product should be satisfying. According to the result of this comparison, the deteriorated status of each part which is a component of the product, is evaluated using product model and behavior-mechanism relation tables. The final output of this evaluation system is the predicted deformation value for each geometric element of the parts.

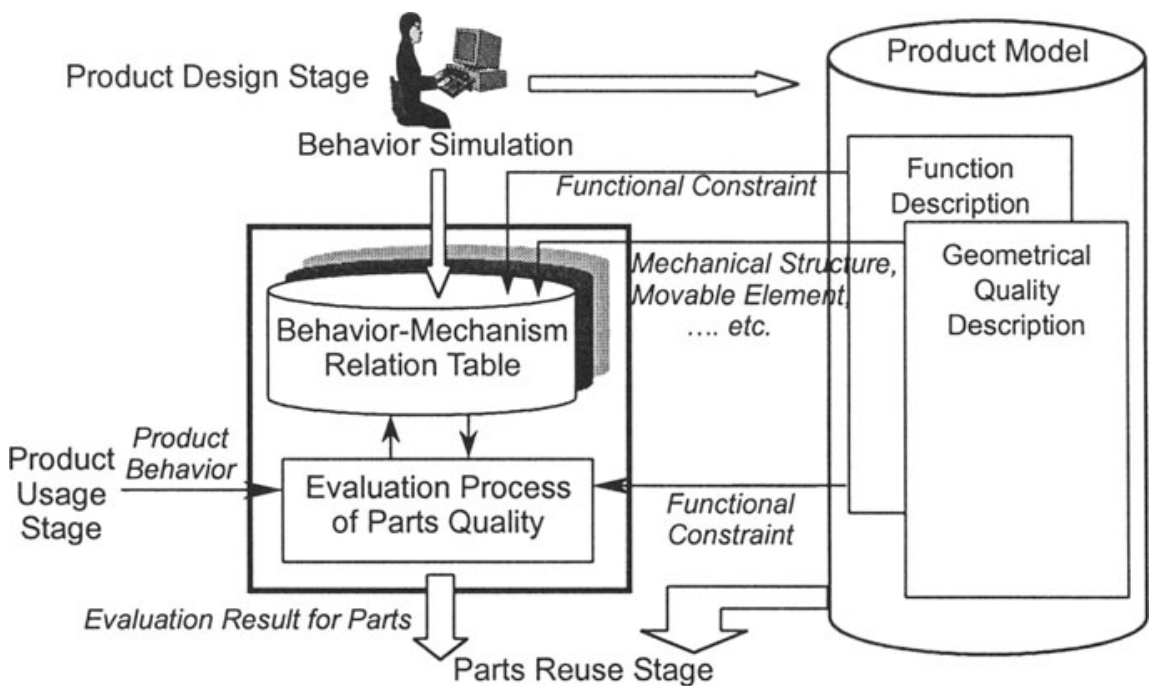

Figure 5. Evaluation process for geometrical quality of used-parts

\subsection{Preparation of the behavior-mechanism relation}

In the preparation process which will be included in the product design 
stage, each set of deteriorated status given by the behavior-mechanism relation tables are tried with the behavior simulation as shown in Figure 6.

First the behavior-mechanism relation table is generated with an entry corresponding to each functional constraint. Then, by looking up the mechanical structure description in the product model, a corresponding movable element is found.

In the behavior simulation of the product, the value of the corresponding geometrical-relation constraint in the movable element description is interpreted as a dimension of the clearance between parts. By superimposing the result of behavior simulation, the effect of that set of deteriorated status on the behavior can be calculated as the threshold value of the corresponding predicted geometrical accuracy. These results are added to the behaviormechanism relation table based on the result calculated by product behavior simulator.

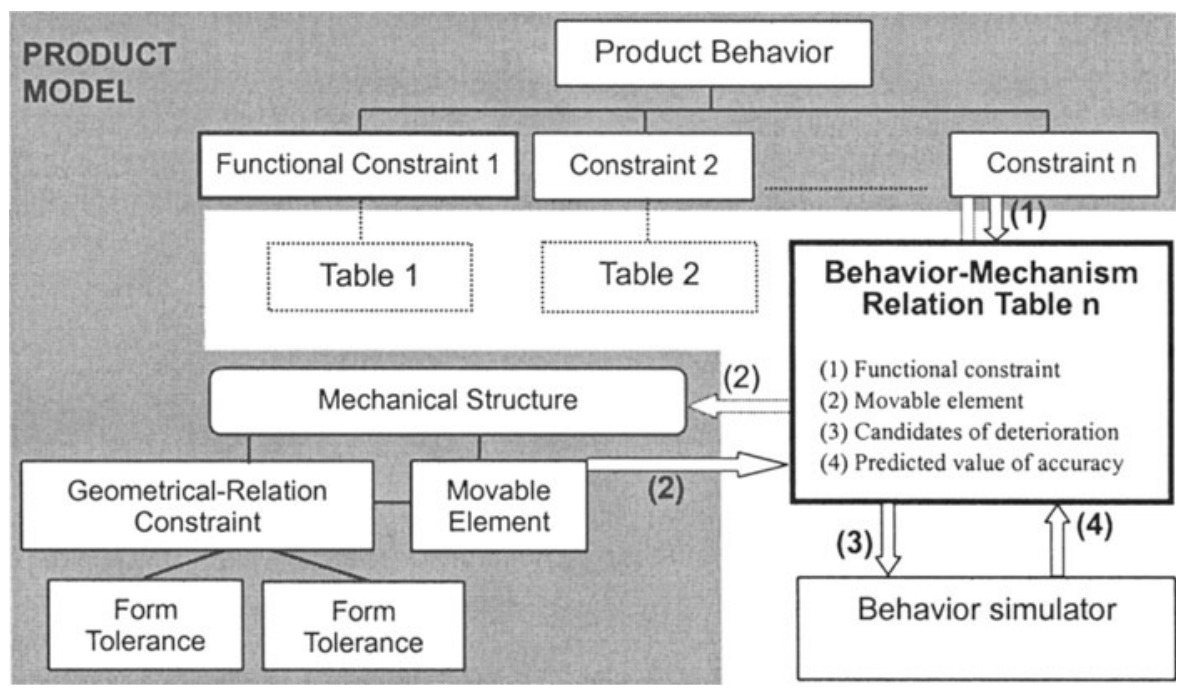

Figure 6. Preparation of the behavior-mechanism relation table

\subsection{Evaluation process}

Evaluation process of the geometrically deteriorated status of the parts is executed using product model and behavior-mechanism relation tables. Figure 7. shows the procedure of the evaluation.

Usage history is also added to the product model during the product use stage. This history mainly describes the functional status of the product corresponding to the functional requirement on the behavior-mechanism relation table. For the evaluation, as the first step the usage history is compared with the threshold value of each functional requirement on the 
behavior-mechanism relation table.

If a functional requirement to be achieved is found to be satisfied from the usage history, the behavior-mechanism relation tables corresponding to the functional requirements can be selected for evaluating the deteriorated status of the related movable elements. When several behavior-mechanism relation tables are selected, tables are overlapped to determine whether the same movable element are related or not.

Using this methodology, the deteriorated status for each movable element is estimated. Then in each movable element, the deformation is assigned to the form tolerance of each geometric element involved in the movable element. The relationship between geometrical-relation constraint and form tolerance is used for this assignment.

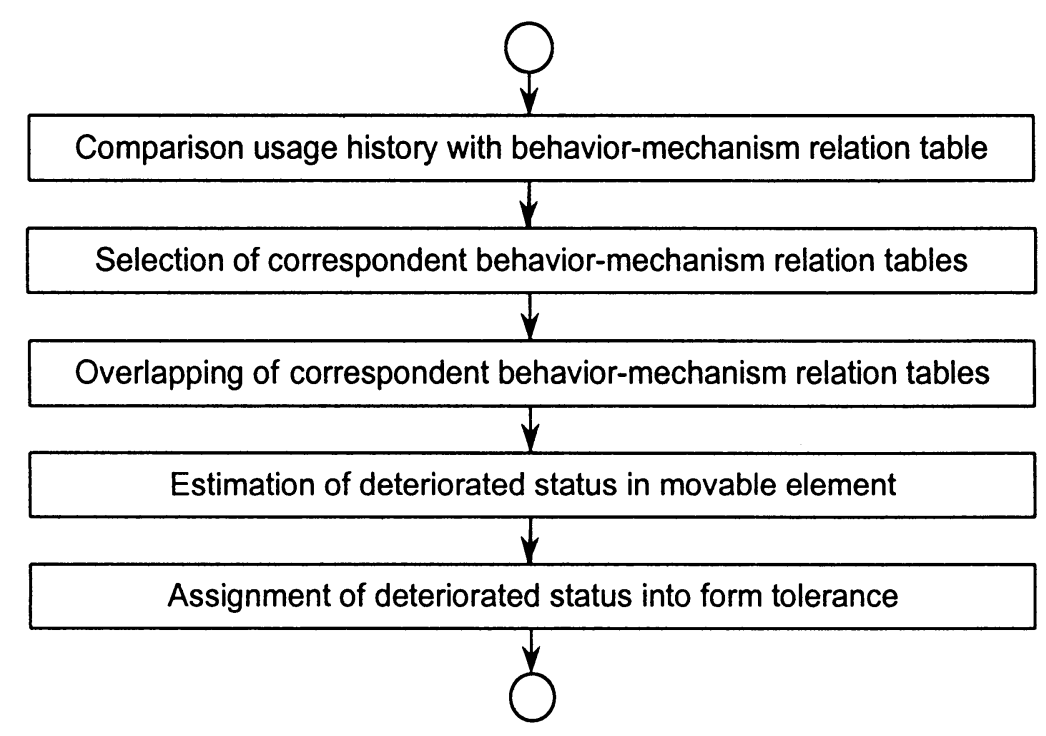

Figure 7. Evaluation process of used-parts quality

\section{EXAMPLE}

The proposed evaluation method is applied to a slider-crank mechanism. Figure 8 shows the mechanism (left) and solid model (right) of the example model.

Based on this solid model, both form tolerances and geometrical-relation constraints are added. Figure 9 shows the part of the geometrical quality description for this example model. In this example, a hinge element class is defined between the cylindrical shaft of part 1 and the cylindrical hole of part2. By repeatedly generating geometrical quality descriptions for all parts, 
the mechanical structure class can be defined as shown in figure 11.

Then, a behavior-mechanism relation table is generated based on the mechanical structure and a functional requirement as shown in Figure 10. In this case, the positional error of the evaluation point on part3 is supposed to be the requirement. As there are three movable elements involved in the mechanical structure class, seven possible sets of deteriorated states are given for the behavior simulation. Then each possible set is checked in the calculation process. In this process, behavior simulation is executed in order to estimate the maximum deformation value for each case. After calculating all cases, the maximum deformation value for each movable element can be detected. Here, ADAMS which was developed by Mechanical Dynamics, Inc., is used as a behavior simulator.

In the evaluation process, if this function requirement is not satisfied, the estimated deformations are assigned to the form tolerance of each geometric element. In this example, the clearance of the above-mentioned hinge element is assigned to the cylindricities of the shaft and hole. As a result, the change of cylindricity of both parts is evaluated as the true cause for the change of behavior.
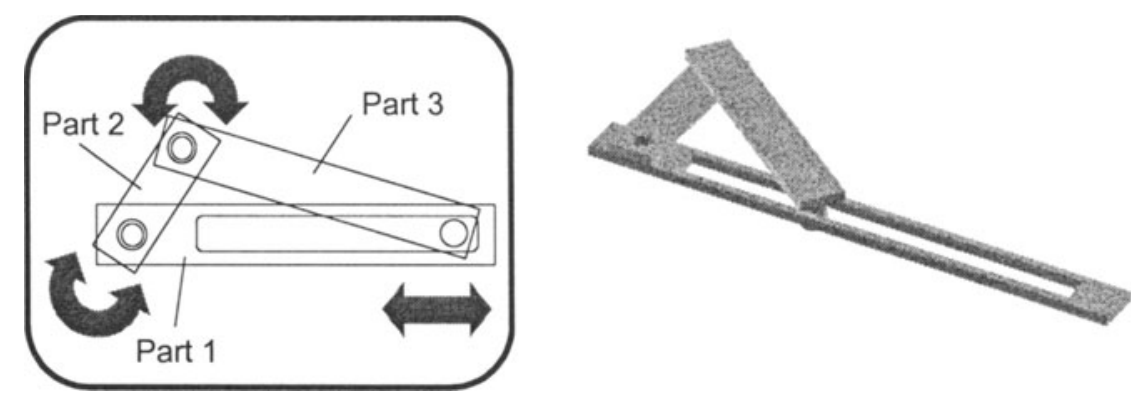

Figure 8. Example parts (slider-crank)

\section{CONCLUSIONS}

In this paper, an evaluation system for used-parts geometrical quality is proposed as one method to integrate the inverse manufacturing process with the traditional forward manufacturing flow. To structure this system, the product model with geometrical quality description and the behaviormechanism relation table are introduced.

The product model with geometrical quality description is provided by expanding the definition of the present geometric tolerance. Therefore, the parts deformation due to deterioration could be interpreted into form 


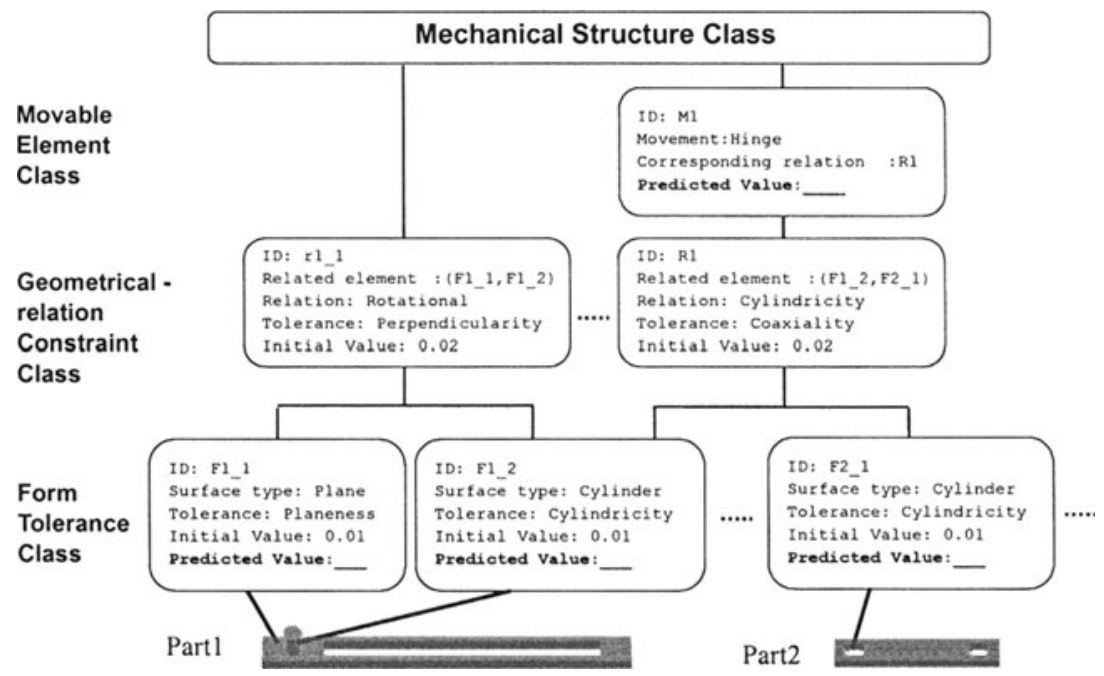

Figure 9. A part of geometrical quality description for example parts

\begin{tabular}{|c|c|c|c|c|}
\hline \multicolumn{2}{|c|}{ Relation Table } & & & \\
\hline \multicolumn{3}{|c|}{ Mechanism Slider-Crank } & & \\
\hline \multicolumn{4}{|c|}{ Functional Requirement: FR01 } & \\
\hline \multicolumn{5}{|c|}{$\begin{array}{l}\text { Error Parameter: Radial Error of Movement } \\
\text { Start Element g12 }\end{array}$} \\
\hline \multicolumn{5}{|c|}{ Evaluated Element g22 } \\
\hline \multicolumn{5}{|c|}{ Thereshold Value: 0.5} \\
\hline hinge! & hinge? & slider & Error & Judge \\
\hline 1.00 & 1.00 & 100 & 1.2135 & 0 \\
\hline 1.00 & $1 \infty$ & 050 & 1.2248 & 0 \\
\hline 100 & 100 & 010 & 1.6987 & 0 \\
\hline 0.50 & 100 & 100 & 0.8410 & 0 \\
\hline 050 & 100 & 050 & 0.8520 & 0 \\
\hline 050 & 100 & 010 & 1.2742 & 0 \\
\hline 010 & 100 & 100 & 0.8136 & 0 \\
\hline 010 & 100 & 050 & 0.5879 & 0 \\
\hline 0.10 & 080 & 100 & 0.7009 & 0 \\
\hline 0.10 & 0.80 & 050 & 0.7565 & 0 \\
\hline 010 & 080 & 010 & 0.7370 & 0 \\
\hline 0.10 & 075 & 100 & 0.4966 & 1 \\
\hline 0.10 & 0.75 & 0.50 & 0.6530 & 0 \\
\hline 1.00 & 0.50 & 1.00 & 0.9685 & 0 \\
\hline 1.00 & 0.50 & 0.50 & 0.8161 & 0 \\
\hline 100 & 0.50 & 0.10 & 1.2648 & 0 \\
\hline 050 & 050 & 1.00 & 0.6550 & 0 \\
\hline 050 & 050 & 050 & 05746 & 0 \\
\hline 050 & 050 & 010 & 08188 & 0 \\
\hline 010 & 050 & 100 & 05976 & 0 \\
\hline
\end{tabular}

Figure 10. A part of generated behavior-mechanism relation table for example parts

accuracy for geometrical element of physical parts. The behavior-mechanism relation table provides a function to connect product behavior with parts deformation.

An example part is applied to the proposed evaluation process from the 
generation of the behavior-mechanism relation table to the prediction of deterioration status. This trial shows the effectiveness of the proposed geometrical quality description and evaluation method using the behaviormechanism relation table.

The output of this evaluation process will be useful for planning of parts reuse. Especially, form accuracy data for geometrical element of parts will be useful for the refurbishment planning phase.

\section{ACKNOWLEDGEMENT}

The authors are grateful to Dr. Udo Graefe of the National Research Council of Canada for his helpful assistance with the writing of this paper in English.

\section{REFERENCES}

[1] Kimura, F. (1997). Inverse Manufacturing: From Products to Services, The $1^{\text {st }}$ Int. Conference Managing Enterprises - Stakeholders, Engineering, Logistics and Achievement (ME-SELA'97).

[2] Hata, T., Kimura, F. and H. Suzuki. (1997). Product Life Cycle Design based on Deterioration Simulation, CIRP $4^{\text {th }}$ Int. Seminar on Life Cycle Engineering, pp 197 -206.

[3] Matsuda, M., and Kimura, F. (1999). A Model Integration Framework for Life Cycle Engineering from the Machining Process Viewpoint, Proceedings of the CIRP $6^{\text {th }}$ International Seminar on Life Cycle Engineering, pp. 332-341.

[4] Suzuki, H., Matsuda, M. and Kimura, F. (2000). Prediction of Used Parts Deterioration Based on A Tolerance Model, Proceedings of the CIRP $33^{\text {rd }}$ International Seminar on Manufacturing Systems, pp. 149-154.

[5] Matsuda, M., Suzuki, H. and Kimura, F. (2001). Tolerance Modeling Using The Feature Based Product Model for Prediction of Used Parts Deterioration, Proceedings of International IIFIP Conference Feature Modeling in Advanced Design for the Life Cycle Systems FEATS2001, in print. 